\title{
Formats de présentation et complémentarité modale dans les logiciels éducatifs
}

\author{
Françoise POYET \\ Centre de Recherches en Education et Formation des Antilles et de la Guyane \\ IUFM, Services centraux, \\ BP399, 97159 Pointe-à-Pitre Cédex (Guadeloupe) \\ Fpoyet@cgit.com
}

RÉSUMÉ. Les avancées technologiques actuelles permettent aux réalisateurs de logiciels éducatifs d'intégrer plus facilement des messages auditifs et visuels. Cette intégration est souvent le fruit d'une démarche empirique sans réelle réflexion préalable. Pour étudier la complémentarité de messages auditifs et visuels du point de vue des contraintes de fonctionnement de la mémoire humaine [BAD 90] et des théories sur la charge mentale [SWE 91], nous avons réalisé une expérience dans laquelle les sujets devaient mémoriser des informations multimodales ${ }^{1}$. Cette expérience a établi que l'influence des modalités de présentation diffère selon le contenu des messages. Ainsi, des messages longs et détaillés (explications techniques) seront mieux mémorisés dans un format visuel; tandis que des informations courtes (noms), présentées auditivement, amélioreront l'intégration de messages visuels complexes (explications portant sur le fonctionnement du dispositif à mémoriser).

\begin{abstract}
The technical advances of today permit educational software programmers to integrate sound and visual more easily into their software. These improvements are often the result of an empirical approach without previous testing in actual situations. In order to study the complementarity of auditory and visual messages from the point of view of their restrictions on the functioning of the human memory [BAD 90] and cognitive load theory [SWE 91], we have produced an experiment in which the subjects had to memorise multi-modal informations. This experiment established that the influence of the different modalities of presentation differ depending on the contents of messages. Thus, long and detailed messages (technical explanations) will be better memorised in a visual format; whereas short format of information such as names (presented as supplementary information) presented auditively, will improve the integration of complex visual messages (explanations relating to the memory function device).
\end{abstract}

MOTS-CLES : multimédia, multi-modale, format de présentation, mémoire, apprentissage, charge mentale, logiciels éducatifs.

KEY WORDS : multimedia, multi-modal, format of instructions, memory, learning, cognitive load, educational software.

1 Dans l'interaction "personne-machine ", on définira le terme " modale " en référence aux modalités sensorielles des sujets (visuelle et auditive). 


\section{Introduction}

Actuellement, les auteurs de didacticiels ne disposent pas d'indications suffisamment précises pour agencer des supports pédagogiques multimédias ${ }^{2}$. Ce qui les amène à ne pas forcément exploiter les composantes visuelles et auditives des messages à bon escient au regard des ressources cognitives des apprenants. Des travaux sur la charge mentale [SWE 91] montrent que la présentation des informations intervient directement sur la charge liée à la tâche en l'allégeant ou en l'augmentant. Dans certains cas, l'apprenant peut même éprouver des difficultés de compréhension dues à une incompatibilité entre la nature de la tâche à réaliser et le format des informations présentées. Les traitements cognitifs semblent différer selon la manière dont sont combinées les informations entre elles. Dans le cas où celles-ci sont associées et complémentaires, elles sont identifiées comme provenant d'une source unique. A l'inverse, lorsque l'articulation entre elles n'est pas directement compréhensible, les informations sont traitées comme provenant de deux sources différentes; ce qui augmente la charge mentale liée à la tâche.

\section{Problématique et rappels théoriques}

\subsection{Le concept de charge mentale}

Les études sur la charge de travail ont pour origine la prise en compte de problèmes pratiques se posant en situation de travail. Le plus souvent elles ont permis de définir des seuils relatifs au niveau de contrainte exercé lors d'une tâche et se traduisant par une baisse de performances. La charge de travail peut être physiologique, visuelle, thermique ou mentale. C'est dans cette dernière acception que les psychologues ergonomes l'envisagent [SPE 88].

Il n'est pas dans notre intention de discuter du fondement théorique du concept de "charge mentale" mais seulement d'en exposer une présentation générale en considérant deux orientations de recherche : celles de l'ergonomie et de la psychologie.

- Dans le premier cas, la "charge mentale" c'est à dire la charge relative au travail intellectuel ("mental work") [SPE 88] est tributaire des possibilités de traitement de l'information du sujet. On définit un modèle hypothétique selon lequel le sujet est considéré comme "un canal de transmission d'informations muni d'une entrée et d'une sortie". On ne s'intéresse pas aux traitements mis en oeuvre, mais aux flux d'entrée et de sortie. La capacité limitée est alors évaluée par le fait que les informations présentées à l'entrée sont transmises ou non à la sortie.

2 Dans une première acception, le terme média doit être pris au sens étymologique du mot en tant "qu'intermédiaire", "moyen de liaison". Les différents messages véhiculés par les médias informatiques incluent du texte, des graphiques, des images fixes (photographiques) ou animées (vidéo), et du son. 
De ce point de vue, l'analyse du concept de charge mentale se fait à partir de deux critères essentiels : la contrainte et le coût. La contrainte désigne généralement un facteur ou un groupe de facteurs externes à la tâche elle-même (la température, une ambiance bruyante etc..) et le coût est évalué par le niveau d'exigence qui indique une variable caractéristique de la performance attendue ou une norme de production.

- Dans le second cas, on envisage davantage les limites des capacités de maintien et de traitement du sujet. En particulier, le sujet disposerait d'un espace de traitement dont la capacité est à peu près la même pour tout le monde [CHA 73] [TUR 89] [BAD 90]. Cet espace étant utilisé à la fois pour le maintien temporaire de l'information et pour sa transformation, un seuil critique de traitement apparaît donc de manière évidente. Plus l'espace est utilisé par le traitement et moins celui-ci est disponible pour les activités de maintien [RIC 90]. Si la notion de capacité limitée est bien établie, le nombre d'unités distinctes susceptibles d'être maintenues simultanément a fait l'objet de nombreux débats. Pour certains, cette limite serait liée à la taille de la mémoire de travail et serait de l'ordre de $7+/-2$ informations [MIL 56]. Pour d'autres, elle correspond à un seuil de 3 à 5 items rappelés par le sujet à partir d'une longue série [BRO 75]. Ces différences proviennent du fait qu'il est difficile de dissocier le maintien de l'information de son traitement. A cet égard, on peut augmenter la capacité de rétention en occupant le système de traitement à des activités qui, comme la révision mentale, favorisent le maintien de l'information en mémoire. En revanche, si le système de traitement est occupé à d'autres activités la capacité de rétention est amoindrie [RIC 90].

\subsection{Charge mentale et mémoire de travail}

La mémoire humaine occupe une place centrale au sein des activités cognitives des sujets car elle est sollicitée en permanence : que ce soit lors de la prise d'informations, de leur maintien ou de leur traitement pour l'accomplissement de tâches simples ou complexes. Il n'est donc pas étonnant que les limites de la capacité mnémonique du sujet interviennent directement sur sa charge mentale à cause des contraintes qu'elle exerce. Selon le modèle de Baddeley [BAD 86 ; 90], la mémoire de travail se compose de l'unité de contrôle (ou administrateur) et de deux sous systèmes complémentaires (boucle phonologique ${ }^{3}$ et système visuo-spatial). L'administrateur, la boucle phonologique et le système visuo-spatial interviennent différemment dans le traitement et le maintien des informations en mémoire de travail.

- L'administrateur joue un rôle de supervision des opérations de maintien et de traitement de l'information.

- La boucle phonologique (B.P) est spécialisée dans le traitement du matériel verbal (texte écrit ou oral).

3 Les traitements phonologiques sont les traitements liés aux fondements de la parole, à la base du langage oral ("speech-based"). 
- D'une manière complémentaire à la boucle phonologique, le système visuo-spatial (V.S.S.P.) gère temporairement les informations de nature visuelle et spatiale.

Bien que les deux sous-systèmes B.P. et V.S.S.P. soient spécialisés dans le traitement d'informations de nature différente, on ne peut pas conclure que les matériels verbaux et spatiaux n'entrent jamais en concurrence. Notamment, il peut y avoir interférence entre le maintien d'informations verbales et visuo-spatiales lorsque le matériel verbal renferme des composantes imagées [DEN 89].

\subsection{Charge mentale et format de présentation}

De nombreux auteurs ont montré que le format de présentation des informations joue un rôle non négligeable sur les performances des sujets lors des apprentissages [SWE 90 ; 91]. La prise d'informations est une phase importante lors du déroulement de la tâche car elle organise les informations présentées pour les rendre compréhensibles et facilement intégrables en mémoire de travail. Certains auteurs [KEA 94] [KUL 94] mettent en évidence que lorsque l'on présente des informations textuelles associées à des cartes géographiques, les sujets obtiennent de meilleurs résultats lors du rappel de ces textes. D'autres recherches ont été menées pour étudier les aspects complémentaires des textes et des schémas [VEZ 85] [BE 88]. Il ressort de ces travaux que la relation entre texte et image varie selon la qualité expressive de l'image et l'usage du texte dans cette relation. Au-delà d'un certain seuil d'abstraction, les relations entre texte et image s'inversent. Par exemple, dans le cas des interfaces graphiques, c'est le texte qui oriente la lecture des messages (barre des menus...) et non pas l'image. De nombreuses situations d'apprentissage imposent une importante charge cognitive qui interfère parfois avec l'objectif de la tâche. Et dans certaines situations d'apprentissage ou de résolution de problèmes, la présentation des instructions impose à elle seule une charge importante avant même que le sujet commence la tâche. A cet égard, certaines expériences étudient comment les ressources cognitives sont réparties pendant l'apprentissage [SWE 90] [CHA 91] en établissant des relations complémentaires entre des schémas et des textes. L'objectif de ces travaux est de définir des formats "idéaux" pour réduire la charge mentale relative à la prise d'informations nécessaire à l'accomplissement de la tâche.

\subsection{Qu'est ce qu'un format idéal ? Les expériences de Sweller et Chandler}

Pour Chandler et Sweller [CHA 90], il s'agit d'un format de présentation, appelé format "Intégré ", dans lequel la complémentarité des schémas et des textes aboutit à l'expression d'une même connaissance mettant en oeuvre une représentation commune. A l'inverse, des informations non complémentaires entretiendraient deux systèmes de représentation en parallèle pouvant gêner le traitement de celles-ci. Deux types de formats sont présentés aux sujets : 
- Le format “Intégré " est composé d'un schéma et de textes écrits regroupés en un seul message et associés en complémentarité.

- Dans le format "Conventionnel ", les textes écrits et le schéma sont séparés. Dans ce format, classiquement utilisé dans les manuels scolaires, les textes écrits servent de légende à la figure.

Sweller et Chandler [SWE 91] montrent que des informations textuelles et graphiques associées peuvent aider ou bien entraver le sujet lors de la compréhension des informations présentées. Dans le cas du format Conventionnel, le traitement d'un même message provenant de plusieurs sources différentes non reliées entre elles, accroît la charge mentale du sujet ; ce format impose un double traitement au sujet (légende et schéma). Dans le cas des formats Intégrés, les textes écrits sont situés sur le schéma et expriment des informations complémentaires de celui-ci. La prise d'informations s'opère alors plus facilement et plus rapidement que dans le cas du format Conventionnel. Le format Intégré présente des informations "préparées à être intégrées" étant donné que la mise en relation du schéma et des textes écrits est réalisée par le format lui-même. Le sujet n'a donc pas à effectuer ce traitement supplémentaire; d'où un allégement de la charge mentale liée à la tache.

Néanmoins, ce format n'est efficace que si certaines conditions sont remplies :

- Lorsque le sujet doit maintenir et associer simultanément des informations nombreuses et complexes provenant des deux sources à la fois (textuelle et graphique) c'est à dire lorsque la charge mentale est importante. Il est beaucoup moins efficace lorsque l'intégration mentale des textes et des schémas n'est pas nécessaire à la réalisation de la tâche à effectuer. Dans ce cas, les sujets identifient la source d'information pertinente pour résoudre le problème et font abstraction de la seconde source. Le traitement de l'information émise par une source unique est alors plus rentable que l'utilisation du format Intégré.

- La complémentarité des informations est comprise et mémorisée par le sujet lorsque le lien entre les deux sources (textes et schéma) est explicite. A l'inverse, les sources uniques du format dissocié sont préférables lorsque le lien n'est pas clairement défini.

Dans cette étude, nous examinons l'influence de la modalité sensorielle (visuelle ou auditive) sur la mémorisation d'informations verbales insérées dans un schéma technique complexe.

Découlant des travaux de Sweller et Chandler, nous avons tenu compte d'une certaine forme de complémentarité -schémas et textes- relative au format "Intégré" pour réaliser les formats de présentation de notre expérience. Nos formats de présentation ont été composés de textes associés à un schéma dans lesquels les informations textuelles apportent des informations uniques qui n'entrent pas en redondance avec lui. Etant donné que Sweller et Chandler montrent aussi que des informations détaillées et complexes font perdre de l'efficacité au format "Intégré ", nous avons étudié l'influence de la nature des informations présentées en faisant varier de façon indépendante la modalité de deux types d'informations : labels et explications. Nous n'avons présenté à chaque étudiant qu'une seule version du logiciel ainsi conçu. 
De plus, les travaux de Baddeley [BAD 90] nous ont servi de cadre théorique pour exprimer les informations sous forme auditive et/ou visuelle. Le schéma a été présenté visuellement et les textes ont été exprimés soit auditivement soit visuellement.

\section{L'expérience}

\section{1. Méthode}

Nous avons réalisé un logiciel éducatif relatif à un enseignement de technologie actuellement dispensé au collège (en classe de quatrième) car nous avons souhaité que ce logiciel soit directement transposable en situation réelle de formation. L'enseignement portait sur le fonctionnement d'un distributeur automatique de boissons (voir figure $\mathrm{n}^{\circ} 1$ à la page suivante).

\section{1. 1. Le matériel}

Le schéma du distributeur, auquel étaient associés des textes écrits et/ou des textes auditifs, était présenté au sujet aussi longtemps qu'il le souhaitait. Le sujet pouvait explorer tous les boutons à son rythme et consulter l'information plusieurs fois à sa convenance, quel que soit le format de présentation des informations. Il devenait actif dès la prise d'informations, par désignation avec la souris, il pouvait ouvrir des fenêtres présentant les textes écrits (explications et labels) ou déclencher l'émission des messages auditifs. Pour faire apparaître les labels, le sujet devait pointer sur les différentes parties de l'appareil. Les explications étaient de deux natures : techniques et relatives au fonctionnement de l'appareil (pour les explications, le sujet devait désigner l'icône représentant un livre avec un point d'interrogation). Les consignes d'utilisation des icônes lui étaient précisées dans le logiciel avant de procéder à l'apprentissage du schéma. 


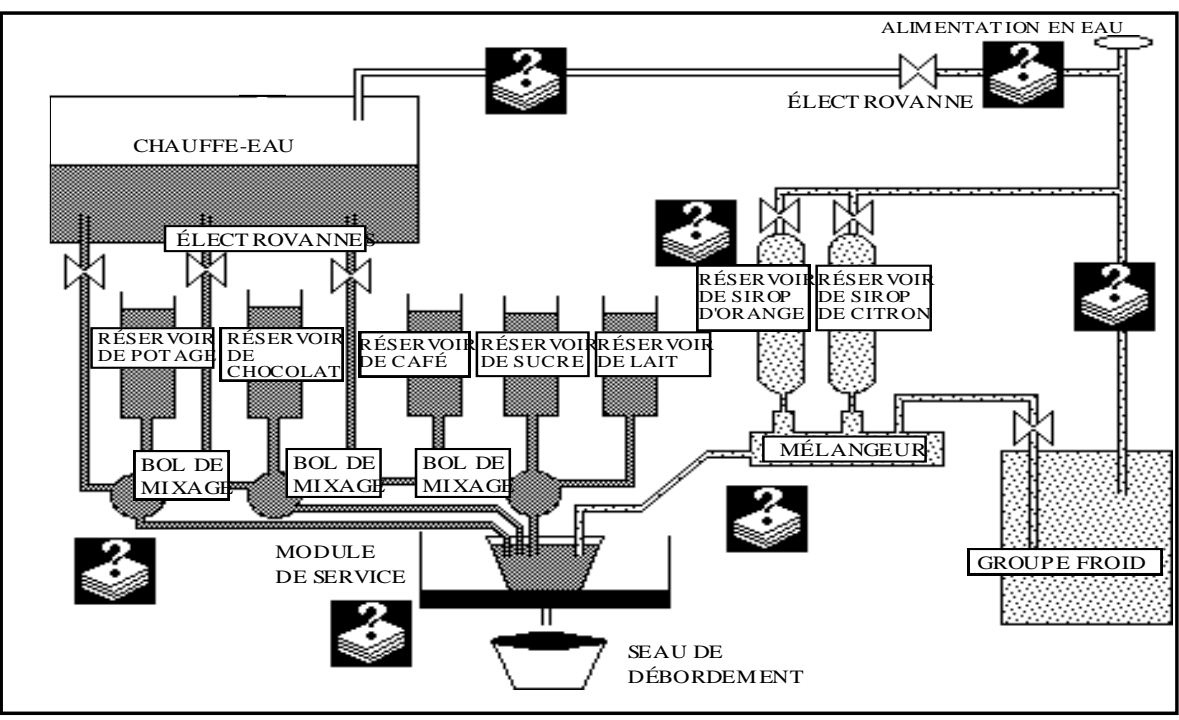

Figure $\mathrm{n}^{\circ} 1$ : Schéma technologique du distributeur automatique de boissons ${ }^{4}$

\section{Les formats de présentation}

1. Dans le format Visuel: Toutes les informations sont présentées sous forme visuelle (schéma et textes). Ce format correspond au format Intégré de Chandler et Sweller.

2. Dans le format Auditif: Tous les textes (labels et explications) sont émis auditivement et se superposent au schéma visuel de l'appareil; quels que soient les textes, ils sont lus par la même personne tout au long du dispositif expérimental.

Le format Mixte revêt deux versions :

3. Dans le format Mixte 1 : Les labels sont auditifs et les explications visuelles;

4. Dans le format Mixte 2 : Les labels sont visuels et les explications auditives.

Les labels et les explications sont présentées en annexe §I et §II.

\subsubsection{Les sujets}

Nous avons travaillé avec une population de quarante-six sujets âgés de 19 à 24 ans (22 ans en moyenne), étudiants en deuxième année universitaire; chaque sujet ne suivait qu'un seul type d'enseignement. L'expérimentation s'est déroulée à Fort de

\footnotetext{
4 Pour simplifier la compréhension du schéma technologique, nous faisons apparaître les labels sur la figure $\mathrm{n}^{\circ} 1$. En fait, lors de la passation des épreuves, les labels n'apparaissent que lors du pointage avec la souris.
} 
France (Martinique). Nous avons constitué quatre groupes de sujets répartis comme suit dans le tableau $\mathrm{n}^{\circ} \mathrm{I}$.

Tableau ${ }^{\circ} \mathrm{I}$ : Répartition des sujets par groupes

\begin{tabular}{|c|c|c|c|c|}
\hline Groupe & 1 & 2 & 3 & 4 \\
\hline Labels & Auditifs & Auditifs & Visuels & Visuels \\
\hline Explications & Visuels & Auditifs & Auditifs & Visuels \\
\hline $\mathrm{N}=46$ & 13 & 10 & 13 & 10 \\
\hline
\end{tabular}

\subsubsection{La procédure}

Le sujet avait pour consigne de :

1.- Mémoriser les labels et les explications.

2.- Comprendre et mémoriser le fonctionnement du distributeur.

Les sujets étaient prévenus que la durée d'apprentissage était illimitée.

Après l'apprentissage du distributeur, on demandait aux sujets de compléter le schéma à l'aide des labels sur un document photocopié. Ensuite, ils devaient répondre à un ensemble de questions portant sur des aspects techniques et sur le fonctionnement du distributeur de boissons à l'aide d'un questionnaire automatisé (voir questionnaire en annexe §III). La restitution des labels faisait l'objet d'une passation antérieure aux questions concernant les explications du schéma. Nous avons supposé que le sujet aurait pu être aidé dans le recouvrement des questions si nous avions changé l'ordre de passation en posant les questions relatives aux explications d'abord.

\subsubsection{Les hypothèses de travail}

\subsubsection{Influence de la modalité des labels}

Lorsque les labels sont présentés dans la modalité auditive, les résultats obtenus lors de la restitution des informations de type Fonctionnement seront meilleurs que lorsque la présentation des labels est visuelle du fait de la complémentarité auditive et visuelle.

En effet, nous pensons que lorsque les informations sont de type technique, les sujets ne font que peu appel aux dénominations de l'appareil pour retrouver l'information. Par contre, lorsque les questions portent sur le fonctionnement de l'appareil, les sujets ont besoin des noms pour raisonner et se construire une représentation du fonctionnement de l'automate. En particulier, les sujets pourraient associer directement les labels auditifs aux labels visuels contenus dans les explications visuelles. Dans ce cas, les traitements phonologiques et visuels seraient complémentaires permettant un allégement de la charge mentale lié à une économie des ressources visuelles. Par conséquent, la présentation concomitante des labels 
auditifs et des explications visuelles devrait favoriser de meilleurs scores lorsqu'il s'agit d'explications portant sur le fonctionnement de l'appareil. Les labels entendus seraient ensuite lus par le sujet dans les explications visuelles et réutilisés pour accéder à la réponse. Dans ce cas, la présentation d'informations identiques provenant de deux sources différentes reliées entre elles par le schéma favoriserait la mémorisation des sujets.

\subsubsection{Influence de la modalité des explications}

Les explications sont des messages longs contenant un nombre important d'informations. Aussi, sont-elles mieux mémorisées sous forme visuelle par comparaison avec le format Auditif des mêmes explications. En effet, la modalité visuelle facilite les relectures et la focalisation sur un ou plusieurs éléments du message si les sujets le souhaitent. Ce qui est plus difficile dans le cas de la présentation auditive des explications qui impose un accès séquentiel aux informations.

\section{2. Présentation des résultats}

Les résultats obtenus à la restitution des labels et aux questions portant sur les explications sont pris comme variables dépendantes dans le plan expérimental $\mathrm{S}<\mathrm{L} 2 * \mathrm{E} 2>$

L2: Modalité des labels (auditive ou visuelle)

E2 : Modalité des explications (auditive ou visuelle)

Les variables dépendantes sont :

V1 : Nombre de labels exacts ; un ou zéro point est attribué selon la nature de la réponse (quinze points est le score maximum).

V2 : Nombre de réponses exactes aux questions. Cette variable totalise vingt points au maximum. Cette mesure se décompose en deux autres mesures :

V2a : Nombre de réponses exactes aux questions techniques. Cette variable totalise dix points au maximum.

$\mathrm{V} 2 \mathrm{~b}$ : Nombre de réponses exactes aux questions portant sur le fonctionnement de l'appareil (dix points au maximum).

\section{2. 1. Influence des modalités des labels sur les performances aux labels}

La moyenne des résultats aux labels a tendance à être meilleure lorsque les labels sont présentés auditivement : 7,57 contre 6,60 sans que cet écart ne soit significatif $\left(\mathrm{F}(1,42)=2,49, \mathrm{~N} \cdot \mathrm{S}^{5}\right)$.

5 N.S. : Non significatif 
10 Sciences et techniques éducatives. Volume 5-n³/1998

Tableau $\mathrm{n}^{\circ}$ II : Table des moyennes aux labels

Effet : Modalités des labels

Variable dépendante : Réponses exactes aux labels

\begin{tabular}{|l|c|c|}
\cline { 2 - 3 } \multicolumn{1}{c|}{} & Moyenne & Ecart-type \\
\hline Labels auditifs & 7,57 & 1,79 \\
\hline Labels visuels & 6,60 & 2,58 \\
\hline
\end{tabular}

Valeur minimale : $0 \quad$ Valeur maximale : 15

2. 2. 2. Influence des modalités des labels sur les performances aux explications de type "Fonctionnement" et "Techniques "

La moyenne des performances aux explications de type "Fonctionnement" est meilleure lorsque les labels sont présentés dans la modalité auditive (6,87 sur 10) par comparaison avec celle qui est obtenue lorsque les labels sont visuels (5,65 sur 10).

L'ANOVA du plan $\mathrm{S}<\mathrm{L} 2 * \mathrm{E} 2>$ permet de mettre en évidence l'effet du facteur "Modalité des labels" sur les performances aux explications de type "Fonctionnement de l'appareil" $(\mathrm{F}(1,42)=7,32, \mathrm{p}<.01)$.

Il n'est pas significatif dans le cas où les explications sont " techniques " $(\mathrm{F}(1,42)=3,03$, N.S $)$.

Tableau $n^{\circ}$ III :Table des moyennes obtenues aux explications de type "Fonctionnement de l'appareil" et "Techniques " (effet modalité des labels)

Effet : Modalités des labels

Variable dépendante : Réponses exactes aux explications “ fonctionnement de l'appareil " et "Techniques"

\begin{tabular}{|l|c|c||c|c|}
\cline { 2 - 5 } \multicolumn{1}{c|}{} & \multicolumn{2}{c|}{$\begin{array}{c}\text { Explications } \\
\text { "Fonctionnement " }\end{array}$} & \multicolumn{2}{c|}{$\begin{array}{c}\text { Explications } \\
\text { Techniques " }\end{array}$} \\
\cline { 2 - 4 } & Moyenne & Ecart-type & Moyenne & Ecart-type \\
\hline Labels auditifs & 6,87 & 1,63 & 6,96 & 1,87 \\
\hline Labels visuels & 5,65 & 1,15 & 6,91 & 1,65 \\
\hline
\end{tabular}

Valeur minimale : 0

Valeur maximale : 10

2. 2. 3. Influence des modalités des explications sur les explications "Techniques " et "Fonctionnement"

Dans le cas des explications “ Techniques", la moyenne des performances aux explications visuelles $(7,57)$ est supérieure à la moyenne des résultats obtenus aux explications auditives (6,30). La présentation visuelle des explications 
"Techniques" facilite donc la mémorisation des sujets par comparaison avec une présentation auditive de celles-ci. On met en évidence l'effet du facteur "Modalité des explications" sur les performances aux "explications techniques" $(\mathrm{F}(1,42)=$ $6,54, \mathrm{p}<.05)$. On ne le vérifie pas pour les explications de type "Fonctionnement" $(\mathrm{F}(1,42)=3,0$, N.S. $)$.

Tableau $\mathrm{n}^{\circ}$ IV : Table des moyennes obtenues aux explications de type "Fonctionnement " et " Techniques " (effet modalité des explications)

Effet : Modalités des explications

Variable dépendante : Réponses exactes aux explications "Techniques" et "Fonctionnement"

\begin{tabular}{|l|c|c|c|c|}
\cline { 2 - 5 } \multicolumn{1}{c|}{} & \multicolumn{2}{c|}{$\begin{array}{c}\text { Explications } \\
\text { "Fonctionnement }\end{array}$} & \multicolumn{2}{c|}{$\begin{array}{c}\text { Explications } \\
\text { Techniques " }\end{array}$} \\
\cline { 2 - 5 } \multicolumn{1}{c|}{} & Moyenne & Ecart-type & Moyenne & Ecart-type \\
\hline $\begin{array}{l}\text { Explications } \\
\text { visuelles }\end{array}$ & 7,57 & 1,75 & 6,70 & 1,59 \\
\hline $\begin{array}{l}\text { Explications } \\
\text { auditives }\end{array}$ & 6,30 & 1,52 & 5,83 & 1,64 \\
\hline
\end{tabular}

Valeur minimale : 0

Valeur maximale : 10

\section{Discussion}

Les résultats de notre expérience montrent deux phénomènes importants :

Les modalités de présentation interviennent différemment sur les performances des sujets selon le contenu des messages.

- La présentation visuelle des explications “Techniques" améliore les performances des sujets. Les propriétés visuelles faciliteraient la mémorisation des informations techniques. Lors du recouvrement des informations en mémoire à long terme, on peut supposer que le sujet peut associer ces informations à des localisations spatiales sur l'écran (à droite, en bas etc...). A la différence des explications de type "Fonctionnement", le rappel des informations "Techniques" ne nécessite pas de transformation particulière. Elles peuvent être maintenues avec la même formulation. Ce qui n'est pas le cas des explications de type "Fonctionnement" faisant davantage appel au raisonnement des sujets. Dans le cas de l'association du schéma avec des textes visuels (de type “ Technique "), la modalité Visuelle pourrait donc apporter des éléments de repérage en favorisant une empreinte visuelle de la localisation de ce type de messages qui serait une clé d'accès lors de leur recouvrement en mémoire à long terme.

- La présentation auditive des labels améliore les performances aux questions de type "Fonctionnement de l'appareil " en jouant sur les complémentarités auditives et visuelles selon le modèle de Baddeley (BP et VSSP). Par exemple, pour savoir comment circule le flux d'eau chaude ou d'eau froide, le sujet est amené à se 
représenter mentalement les flux. On peut donc supposer qu'il effectue nécessairement des traitements visuo-spatiaux pour interpréter le schéma technologique mobilisant à cette fin une partie importante de ses ressources visuo-spatiales. L'intégration de labels auditifs dans une représentation imagée permettrait d'alléger la charge mentale liée aux traitements visuo-spatiaux inhérents à l'élaboration de la représentation mentale. Cette interprétation pourrait être confirmée par les résultats obtenus aux explications “ Techniques " précédemment cités. Ce type d'explications ne nécessite probablement pas les mêmes traitements que les explications "Fonctionnement". On peut faire l'hypothèse qu'il suffirait de maintenir l'information sans la transformer ; par exemple, pour mémoriser le message : "la société est située à Nanterre ", le sujet n'est pas contraint de réfléchir sur le fonctionnement de l'appareil dans sa globalité pour donner la bonne réponse. Les traitements impliqués lors de son raisonnement solliciteraient moins les ressources visuo-spatiales que dans le cas des explications de type "Fonctionnement" étant donné que le sujet n'a pratiquement pas besoin de se remémorer la logique de fonctionnement de l'appareil dans sa globalité.

Dans le prolongement de cette étude et dans la perspective de travaux ultérieurs, différents points théoriques et méthodologiques peuvent être discutés. Pour mesurer l'influence des formats multimodaux sur les performances des sujets, nous avons choisi des épreuves de mémoire en faisant varier différentes catégories de textes : des labels et des explications. Ces catégories ne peuvent pas être considérées comme des indices d'apprentissage car elles ne recouvrent que quelques exemples de messages-types rencontrés dans les documents pédagogiques. Elles peuvent néanmoins constituer des repères quant à la profondeur des traitements mis en oeuvre pour parvenir à la compréhension des textes lors de leur mémorisation.

Certains auteurs [COI 96] définissent le terme " niveau de traitement" comme étant un ensemble organisé d'opérations psychologiques dont la mise en ouvre aboutit à une représentation construite, éventuellement transitoire, relevant de ce niveau. De cette définition, nous retiendrons que la compréhension de messages textuels peut faire appel à l'élaboration de représentations relatives à des niveaux de traitement différents. Les auteurs distinguent six niveaux de traitement:

- graphémique/phonologique

- lexical

- syntaxique

- microstructural

- macrostructural

- modèle de situation

Cette définition est volontairement réductrice car les auteurs ne considèrent pas l'activation de représentations mentales issues de la mémoire à long terme comme un niveau de traitement distinct. Au niveau lexical, il s'agit d'accéder à la signification des mots faisant partie du lexique; dans notre expérience, c'est le cas des dénominations comme " bol de mixage, mélangeur " etc... Les explications de type 
"Technique "6 nécessiteraient quant à elles, la construction d'une forme logique littérale soumise à interprétation sémantique. On pourrait identifier dans cette catégorie de textes, des phrases relevant du niveau d'élaboration microstructural c'est à dire lié à la construction de la séquence de propositions représentant la signification du texte. Sur le plan sémantique, les messages de type "Fonctionnement" semblent nécessiter des niveaux d'élaboration à la fois macrostructuraux et relatifs aux modèles de situation. Rappelons que le niveau macrostructural opère à partir de la microstructure, des buts et des schémas du sujet, pour aboutir à une représentation de l'information condensée à partir des informations du texte. Quant aux modèles de situation, ils correspondent à l'organisation des différents types de textes pour aboutir à une représentation mentale globale tenant compte des aspects co-textuels (liés aux parties du texte venant d'être lues) et les connaissances du sujet (liées au domaine et au contexte). Par exemple, pour mémoriser la phrase suivante :"L'eau provient $d u$ circuit d'alimentation, passe dans le chauffe-eau, puis dans les bols de mixage afin de permettre la fabrication des boissons chaudes ... ", le sujet est amené à se construire une représentation mentale globale du dispositif proche du modèle de situation selon Van Dijk et Kintsch [VAN 83]. A travers ces trois types de messages (labels, explications techniques et fonctionnement), nous pensons avoir sélectionné des catégories de textes particulièrement présents dans les manuels pédagogiques en vue d'étudier l'influence de leur présentation sur la charge mentale liée à la tâche. Nous pourrions donc poursuivre ce travail en envisageant d'autres catégories de textes et en analysant l'influence de la multimodalité sur la compréhension et la mémorisation de ces messages. Parmi ces catégories, nous pourrions retenir: les messages récapitulatifs (titres, résumés, synthèses, annotations, sigles etc...), les descriptions, les exposés, les récits, etc...

Enfin, dans l'expérience présentée, le matériel utilisé a été extrait d'un manuel scolaire s'adressant à des élèves scolarisés en classe de $4{ }^{\text {ème }}$ technologique. Ce cours présentait l'attrait de ne demander aucun pré-requis pour le public d'adultes auquel nous l'avons présenté. Il serait intéressant de renouveler l'expérience auprès d'élèves de $4^{\text {ème }}$ technologique pour observer ou non d'éventuels écarts.

\section{Conclusion}

Notre expérience a répondu à la plupart des objectifs fixés. Elle nous a permis de montrer que les modalités n'ont pas la même influence sur les performances des sujets selon la nature des informations transmises (labels versus explications). De plus, au sein des explications "techniques" (description des objets) ou de type " fonctionnement" (élaboration d'un modèle de situation), nous avons établi que ces modalités vont se comporter encore différemment. Par conséquent, il paraît délicat, à l'issue de nos résultats de valider un modèle d'association schéma et parole tel que nous l'aurions souhaité, mais nous pouvons néanmoins suggérer que certaines

6 A titre d'exemple : “ Les sirops utilisés sont de marque Texal GU et sont référencés H410 pour l'orange et H411 pour le citron", 
complémentarités existent et passent par la prise en compte du contenu des messages présentés.

Ainsi, ces résultats nous permettent de formuler quelques recommandations à l'intention des développeurs de logiciels éducatifs quant à l'utilisation du multimédia.

- La présentation visuelle de textes longs et encombrés de détails améliore la mémorisation des sujets par comparaison avec une présentation auditive.

- Lorsque le sujet est amené à fournir un raisonnement complexe sur l'ensemble d'un dispositif, la présentation auditive de certaines informations (labels) améliore la mémorisation de messages explicatifs présentés visuellement.

En conséquence, ces expériences ouvrent des perspectives intéressantes dans la mesure où d'autres types de messages tout aussi présents dans les manuels pédagogiques pourraient être pris en considération.

\section{Remerciements}

L'auteur tient à remercier sincèrement Jean-François ROUET, chercheur au laboratoire "langage et Communication ” ERS CNRS 591 (Poitiers), pour ses précieux conseils. 


\section{Bibliographie}

[BAD 74] BADDELEY A.D et HITCH G., “Working memory ” In G.A. Bower (Ed.). Recent advances in learning and motivation, Vol.8, New-York :Academic press., 1974.

[BAD 90] BADDELEY A.D., Human memory : Theory and Practice, Hove : L.E.A, 1990.

[BER 88] BERTIN J., La graphique et le traitement graphique de l'information, Paris : Flammarion, coll. nouvelle bibliothèque scientifique, 1988.

[BET 88] BETRANCOURT M. et BISSERET A., “Interaction texte / figure : effet de leur disposition spatiale relative sur l'apprentissage ", Rapport de recherche non publié, 1992, INRIA-IRIMAG.

[BRE 93] BREWSTER S.A., WRIGHT P.C., and EDWARDS A.D.N., “ An evaluation of earcons for use in auditory human-computer interfaces ", Actes du congrès INTERCHI'93, 24-25 avril 93 : ACM, p. 222-227.

[BRO 75] BROADBENT D.E., "The Magic Number Seven After Fifteen Years", in KENNEDY A., WILKES A.(Ed) Studies in Long Term Memory, New-York, Wiley, 1975.

[BRU 92] BRUNER J; ... car la culture donne forme à l'esprit : de la révolution cognitive à la psychologie culturelle, Paris, édition Eshel, publié sous le titre Acts of meanings, Harvard University Press, 1990.

[BUX 89] BUXTON W., "Introduction to this special issue on nonspeech audio", Human-Computer Interaction, 4, 1989, p. 1-9.

[CHA 91] CHANDLER P. et SWELLER J., " Cognitive Load Theory and the format of Instruction ”, Cognition and Instruction, 8 (4), 1991, p. 293-332.

[CHA 73] CHASE W.G. and SIMON H.A., " Perception in chess ”, Cognitive psychology, 4, 1973, p.55-81.

[COI 96] COIRIER P., GAONAC'H D., PASSERAULT J.M., Psycholinguistique textuelle, Liège, Armand-Colin, 1996.

[DEN 82] DENIS M., “ Représentation imagée et résolution de problèmes ”, Revue française de pédagogie, $\mathrm{N}^{\circ} 60,1982$, p. 19-29.

[FAY 92] FAYOL M., GOMBERT J. E., LECOCQ P., SPRENGER-CHAROLLES L., ZAGAR D, Psychologie cognitive de la lecture, Paris, PUF, 1992.

[GOL 91] GOLDMAN S. R., "On the Derivation of Instructional Applications From Cognitive Theories : Commentary on Chandler and Sweller ", Cognition and Instruction. 8(4), 1991, p. 333-342. 
16 Sciences et techniques éducatives. Volume 5-n³/1998

[HUL 87] HULME C., “ The effects of acoustic similarity on memory in children: a comparison between visual and auditory presentation ", Applied Cognitive Psychology, 1, 1987, p.45-51.

[KUL 94] KULAVY R.W., STOCK W.A. et KEALY W.A., “ How geographic maps increase recall of instructionnal text ", ETR \& D, Vol.41, 1994, p. 47-62.

[LeCO 92] LeCOMPTE DENNY C., “In search of a strong visual recency effect ”, Memory and Cognition, 20(5), 1992, p .563-572.

[MILL 56] MILLER G.M., “ The magical number seven, plus ou minus two : some limits on our capacity for processing information ”, Psychological Bulletin, 63, 1956, p. 81-97

[PER 88] PERRUCHET P., Les automatismes cognitifs, Liège, Mardaga (Ed), 1988.

[RIC 90] RICHARD J.F., Les activités mentales : comprendre, raisonner, trouver des solutions, Paris, Armand Colin, 1990.

[SPE 88] SPERANDIO J.C., L'ergonomie du travail mental, Paris, Masson, 1988.

[SWE 1991] SWELLER J. and CHANDLER P., "Evidence for Cognitive Load Theory", Cognition and Instruction, 8(4), 1991, p. 351-362.

[SWE 1990] SWELLER J., CHANDLER P., TIERNEY P. and COOPER M., " Cognitive Load as a Factor in the Structuring of Technical Materiel ", Journal of Experimental Psychology : General, Vol. 119, N², 1990, p. 176-192.

[VAN 83] VAN DIJK T.A., KINTSCH W., Strategies of discourse comprehension, New York, Academic Press, 1983.

\section{Biographie}

Françoise POYET est Docteur en psychologie et Maître de Conférences Associé en Sciences de l'Education à l'université des Antilles et de la Guyane (Martinique). Elle y enseigne la psychologie de l'éducation et les technologies éducatives. Membre du Centre de Recherches en Education et Formation des Antilles et de la Guyane, elle s'intéresse à l'usage des Technologies de l'Information et de la Communication (T.I.C.) en pédagogie. En particulier, elle étudie leur influence sur les activités cognitives des sujets en situation d'apprentissage par ordinateur. De plus, depuis deux ans, elle accompagne un projet d'innovation technologique en formation à la Chambre de Commerce et d'Industrie de la Martinique. La mise en place de deux centres de formation multimédias a permis de poser d'authentiques problèmes d'ingénierie éducative. 


\title{
Presentation format and modal
}

\section{complementarity in educational software}

\author{
Annexe I \\ 1. Présentation des labels \\ - réservoir de potage \\ - réservoir de chocolat \\ - réservoir de sucre \\ - chauffe-eau \\ - bol de mixage \\ - réservoir de café \\ - mélangeur \\ - réservoir de lait \\ - alimentation en eau \\ - réservoir de sirop de citron \\ - électrovannes \\ - réservoir de sirop d'orange \\ - seau de débordement \\ - groupe froid \\ - module de service
}

\section{Présentation des explications}

Message 1 "Le rôle de l'alimentation en eau "."Elle permet de remplir le groupe-froid ainsi que le chauffe-eau et de les maintenir sous pression par le jeu des électrovannes.

L'alimentation en eau est raccordée à l'arrivée d'eau par un tube de cuivre ou de plastique de 6 millimètres par 8 comportant un robinet d'arrêt".

Message 2 "Définition du circuit d'eau chaude". "L'eau provient du circuit d'alimentation, passe dans le chauffe-eau, puis dans les bols de mixage afin de permettre la fabrication des boissons chaudes (chocolat, café et potage) (Partie gauche du schéma)".

Message 3 "Le service des boissons". "Après que la poudre soit brassée avec l'eau chaude dans les bols de mixage, la boisson est servie dans un gobelet se trouvant dans le module de service. Les eaux usées sont alors récupérées dans le seau de débordement.

Le distributeur automatique de gobelets en carton contient 600 verres au maximum."

Message 4 "Définition du circuit d'eau froide". "L'eau part du circuit d'alimentation, passe dans le groupe-froid pour être rafraîchie et remonte jusqu'à la 
partie "mélangeur", afin de fabriquer des boissons au citron ou à l'orange (Partie droite du schéma).

Les sirops utilisés sont de marque Texal GU et sont référencés $H 410$ pour l'orange et H411 pour le citron.

Message 5 "Fabrication des boissons froides". "Le mélangeur permet l'incorporation du sirop de citron ou d'orange avec l'eau rafraîchie dans le groupe-froid.

Il maintient la température de l'eau à 7 ou 8 degrés centigrades".

Message 6 "Le rôle des bols de mixage". "Les bols de mixage permettent le brassage de plusieurs ingrédients au choix (poudre, eau, sucre...) dans des récipients spécifiques à la production de chacune des boissons chaudes.

Les bols mixent les ingrédients avec une vitesse de 8000 tours par minute pour le chocolat et le potage et avec une vitesse de 3000 tours par minute pour le café, avant de laisser la boisson descendre dans le gobelet".

Message 7 "Le rôle des électrovannes". "Les vannes sont pilotées automatiquement lors de la sélection de la boisson par le client. Elles commandent l'ouverture ou la fermeture des circuits d'eau et de poudre.

Pour toute réparation concernant les électrovannes,

vous devez vous adresser à la société

SIAPA, 92 rue Petit, 92000 Nanterre".

Dans les messages, les parties en italique présentent les informations "techniques" pour les différencier de celles concernant le fonctionnement de l'appareil. Nous introduisons cette différence ici afin de faciliter la compréhension du lecteur car dans la version expérimentale nous n'avons pas observé de différences au sein des messages visuels.

\section{Présentation du questionnaire portant sur les Explications}

Propositions relatives au rappel des explications portant sur le "fonctionnement de l'appareil':

1. Le mélangeur permet le mélange du sucre dans le café.

2. Il est possible de supprimer le sucre dans le café.

3. Le potage et le chocolat sont préparés dans le même bol de mixage.

4. L'eau contenue dans le groupe froid provient du circuit d'alimentation en eau.

5. La distribution d'eau est pilotée par des électrovannes.

6. Le remplissage d'eau du groupe froid est piloté par une électrovanne.

7. Si l'on ferme la vanne située près de l'alimentation en eau, la boisson au citron n'est plus disponible. 
8. L'eau contenue dans le chauffe-eau ne provient pas du groupe froid.

9. Les poudres de chocolat et de lait sont brassées dans le même bol de mixage.

10. Le chauffe-eau n'est pas sous pression permanente.

\section{Propositions relatives au rappel des explications "techniques":}

11. Le chauffe eau absorbe une puissance électrique de $12350 \mathrm{~W}$.

12. Le nom de la société de réparation des électrovannes est SIMON

13. Le seau de débordement sert à recevoir les effluents provenant du module de service.

14. L'alimentation en eau est raccordée par un tube de cuivre ou de plastique comportant un robinet d'arrêt.

15. Le groupe froid maintient la température de l'eau en dessous de 7 degrés.

16. Le sirop d'orange est de marque Texal GU et de référence H411.

17. Le chauffe-eau maintient la température de l'eau à 35 degrés.

18. Le distributeur automatique de gobelets peut contenir plus de 600 verres.

19. La société de réparation des électrovannes se situe à Nanterre.

20. La section du tuyau de raccordement avec l'alimentation en eau est de 6 millimètres par 8 . 